\title{
BMJ Open Autologous transplantation of umbilical cord blood-derived cells in extreme preterm infants: protocol for a safety and feasibility study
}

Atul Malhotra (10 ,1,2,3 Iona Novak, ${ }^{4}$ Suzanne Lee Miller, ${ }^{3,5}$ Graham Jenkin ${ }^{3,5}$

To cite: Malhotra A, Novak I, Miller SL, et al. Autologous transplantation of umbilical cord blood-derived cells in extreme preterm infants: protocol for a safety and feasibility study. BMJ Open 2020;10:e036065. doi:10.1136/ bmjopen-2019-036065

- Prepublication history for this paper is available online. To view these files, please visit the journal online (http://dx.doi. org/10.1136/bmjopen-2019036065).

Received 28 November 2019 Revised 31 January 2020 Accepted 09 April 2020

D Check for updates

(c) Author(s) (or their employer(s)) 2020. Re-use permitted under CC BY-NC. No commercial re-use. See rights and permissions. Published by BMJ.

${ }^{1}$ Department of Paediatrics, Monash University, Melbourne, Victoria, Australia

${ }^{2}$ Monash Newborn, Monash Children's Hospital, Melbourne, Victoria, Australia

${ }^{3}$ The Ritchie Centre, Hudson Institute of Medical Research, Melbourne, Victoria, Australia ${ }^{4}$ Cerebral Palsy Alliance, Sydney, New South Wales, Australia

${ }^{5}$ Department of Obstetrics and Gynaecology, Monash University, Melbourne, Victoria, Australia

Correspondence to

Dr Atul Malhotra;

atul.malhotra@monash.edu

\section{ABSTRACT}

Introduction Preterm brain injury continues to be an important complication of preterm birth, especially in extremely premature infants. Umbilical cord bloodderived cells (UCBCs) are increasingly being evaluated for their neuroprotective and neuroreparative properties in preclinical and clinical studies. There remains a paucity of information on the feasibility and safety of autologous UCBC transplantation in extremely premature infants. Methods and analysis A single centre safety and feasibility study in preterm babies born before 28 weeks gestation. Cord blood will be collected after birth and if sufficient blood is obtained, UCB mononuclear cells will be harvested from the cord blood, characterised and stored. After excluding infants who have already suffered severe preterm brain injury, based on cranial ultrasounds in first week of life, preterm infants will be infused with autologous UCBCs via the intravenous route at a dose of $25-50$ million UCBCs $/ \mathrm{kg}$ body weight of live cells, with the cell number being the maximum available up to 50 million cells $/ \mathrm{kg}$. A minimum of 20 infants will be administered autologous UCBCs. Primary outcomes will include feasibility and safety. Feasibility will be determined by access to sufficient cord blood at collection and UCBCs following processing. Safety will be determined by lack of adverse events directly related to autologous UCBC administration in the first few days after cell administration. Secondary outcomes studied will include neonatal and neurodevelopmental morbidities till 2 years of life. Additional outcomes will include cell characteristics of all collected cord blood, and cytokine responses to cell administration in transplanted infants till 36 weeks' corrected age.

Ethics and dissemination Monash Health Human Research Ethics Committee approved this study in December 2019. Recruitment is to commence in July 2020 and is expected to take around 12 months. The findings of this study will be disseminated via peer-reviewed journals and at conferences.

Trial registration number ACTRN12619001637134.

\section{INTRODUCTION}

More than 3000 very preterm infants (born less than 32 weeks' gestation) are born every year in Australia and around $7 \%$ of them go on to develop cerebral palsy (CP). A higher
Strengths and limitations of this study

- A world first study to evaluate the safety and feasibility of autologous umbilical cord blood collection and mononuclear cell infusion in extreme premature infants.

- A study translating preclinical advances in cellbased therapies for perinatal brain injury to the clinical arena.

- Assessment of preterm cord blood cell characteristics and cytokine profiling of response to cell transplantation that may inform mechanisms of action of umbilical cord blood-derived cells and the design of future efficacy trials.

- Given the phase 1 nature of the study, there are no prospective controls or randomisation.

proportion of them develop other adverse neurodevelopment outcomes, including developmental delays, cognitive deficits and behavioural disorders. Preterm brain injury (mostly intraventricular haemorrhage (IVH) and periventricular leukomalacia) complicates the neonatal course of very preterm infants (especially extremely preterm infants; those born before 28 weeks' gestation). ${ }^{1}$ Most preterm infants who suffer severe preterm brain injury (severe IVH, cystic periventricular leukomalacia) in the neonatal period have very poor prognosis, with a proportion of them dying during the neonatal period, needing shunt surgery for post haemorrhagic hydrocephalus and, in those who survive, many go on to suffer from adverse long-term neurodevelopment outcomes, including CP. Extreme preterm infants with less severe forms of brain injury in the neonatal period are also at risk of adverse neurodevelopment. ${ }^{2}$ Advances in neonatal care have substantially improved survival in very preterm and extremely preterm infants with brain injury, but no early treatment is currently available for preterm brain injury. 


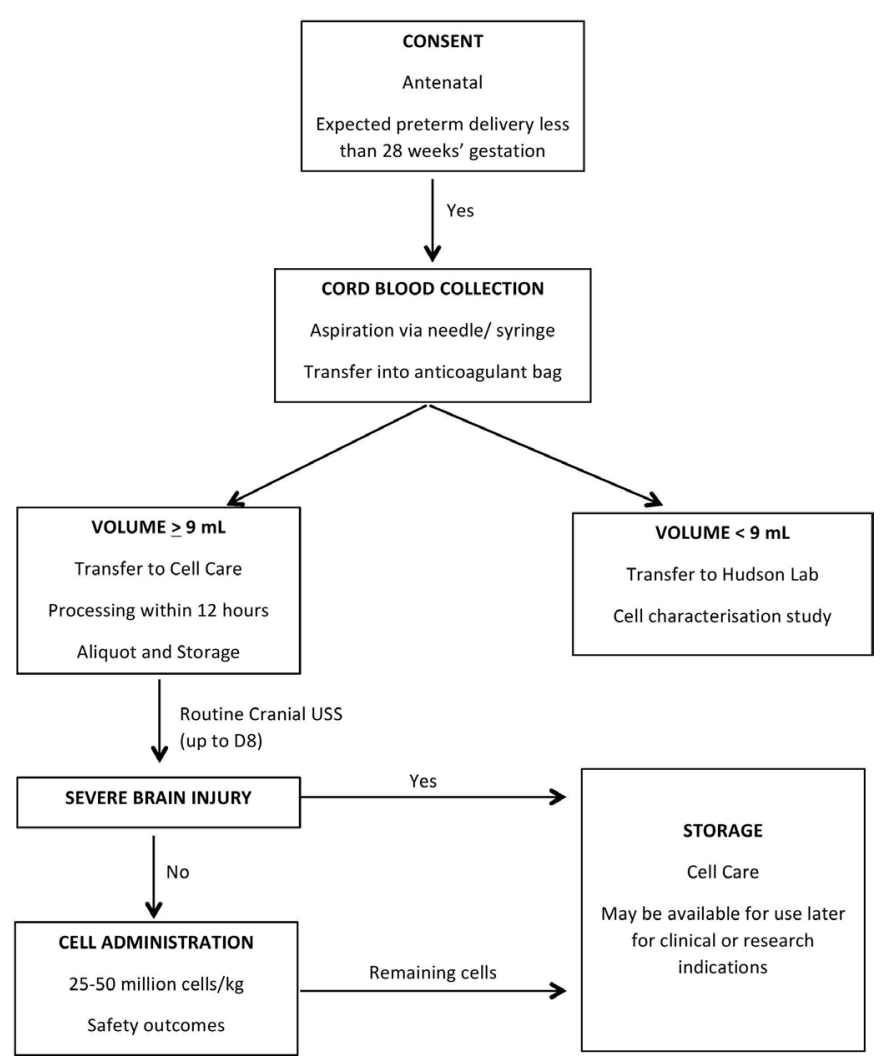

Figure 1 Participant flow.

Cell therapies are increasingly being evaluated for neuroprotection and neuroregeneration in young children, ${ }^{3-6}$ including one current trial using sibling umbilical cord blood (UCB) in young children with confirmed $\mathrm{CP}$ in Australia. Further trials are proposed. There is now substantial preclinical evidence that administration of UCB-derived mononuclear cells in the early neonatal period reduces perinatal brain injury and prevents the progression of neuropathology and CP in the long term. Umbilical cord blood-derived cells (UCBCs) have been shown to be neuroprotective for the preterm brain when administered in preclinical models of preterm brain injury. ${ }^{7-10}$ More recently, we have shown that a single dose of UCBC therapy also delivered improvements in longterm behavioural outcomes in a rat model of neonatal hypoxic ischaemic injury. ${ }^{11} \mathrm{UCB}$ can be easily and safely collected at birth, leading to a high cell yield which contains a wide variety of stem and progenitor cells that have been shown to mediate positive benefits on a variety of neurological cells, including glial cells, neurons and cells that maintain the blood brain barrier (BBB). ${ }^{31012-14}$ These, and other, studies show that the neuroprotective and neuroreparative benefits of early UCBC therapy for the developing brain are likely mediated by their antiapoptotic, anti-inflammatory, pro-angiogenic, neurogenic, antioxidant and BBB protective mechanisms. ${ }^{5-10}$ Most of these studies have used the term cord bloodderived cells, but we have also investigated the neuroprotective/neuroreparative characteristics of preterm UCBCs. ${ }^{715}$ There are only two published clinical studies, which have been conducted, in preterm infants using autologous UCBCs, but the gestation age of included infants was greater than 28 weeks. ${ }^{16} 17$

We hypothesise that in extremely premature infants, (1) autologous UCBC collection at birth will be feasible and the (2) intravenous administration of UCB-derived stem cells in the neonatal period will be safe. The specific aims of this study, thus, are to test the feasibility of collection of sufficient UCBC from cord blood; and safety of autologous intravenous UCBC administration in extremely preterm infants during the neonatal period.

\section{METHODS AND ANALYSIS \\ Design \\ Phase 1 , single centre feasibility and safety study.}

\section{Setting}

Monash Newborn, Monash Children's Hospital, Monash Health.

\section{Participants}

Extreme preterm infants born before 28 completed weeks of gestation (up to $27^{+6}$ weeks).

\section{Sample size}

We aim to administer UCBCs to at least 20 extremely preterm infants at the dose range detailed below. The sample size is consistent with other phase 1 UCBC trials conducted in newborn infants. ${ }^{3}{ }^{36}$ The number of recruited infants and cord blood collections will exceed 20 as we may not have enough cord blood collected after birth, or may not have enough UCBCs available for administration after processing of the cord blood. Further, some infants may be excluded after cord blood collection as per exclusion criteria below. See participant flow in figure 1.

\section{Cord blood collection}

\section{Cord blood collection and processing}

UCB will be collected from all eligible extreme preterm infants of parents who have consented. Size appropriate needle (18-21 G) and collection containers/bags (Macopharma, Tourcoing, France) will be used to maximise the volume of cord blood that can be obtained. Minimum cord blood collection volume for inclusion in the trial will be $9 \mathrm{~mL}$. Infants with collected cord blood volume of less than $9 \mathrm{~mL}$ will be excluded from the trial, but their cord blood will be retained and its characteristics investigated as part of a substudy. For all other infants, the collected cord blood $(\geq 9 \mathrm{~mL})$ will be processed according to standard cord blood processing procedures, aliquoted and cryopreserved. $^{18} 19$

\section{Storage and release}

An aliquot containing sufficient numbers of UCBCs, according to weight of the preterm infant, required for early administration (see dose below) will be stored. Any excess UCBCs will compose further aliquot(s) that will 
be stored separately for potential subsequent use, not comprising part of the current trial. Cell Care (Heatherton, Victoria, Australia), a TGA (Therapeutics Goods Administration)-accredited cord blood bank, will be responsible for collection, processing, storage of all cord blood and release of UCBCs for autologous administration to the preterm infants. Standard testing for microbes, cell viability and DNA matching of UCBC to the preterm infant's DNA will be performed before release of UCBCs. Criteria for product release will include: free of microbial contamination after 7 days of culture, cell viability $>70 \%$ as determined by trypan blue exclusion at the time of cryopreservation and following prior to administration, after thawing and washing of cryopreserved cells.

\section{Thawing and infusion preparation}

On the day of infusion, UCBCs will be retrieved from liquid nitrogen storage, and, once product release criteria are met, the frozen UCBC aliquot will be transported to Monash Health. Cells will be thawed using a pre-warmed bead bath for approximately $2 \mathrm{~min}$. The UCBCs will be washed with dextrose/albumin and centrifuged at $350 \mathrm{~g}$ for $5 \mathrm{~min}$ prior to resuspension in dextrose/albumin, viability testing and counting of live cell number and dilution to the final desired concentration. The UCBCs will be suspended at a concentration $10 \%$ greater than the desired concentration to allow for cell loss. Volume of cell infusion prepared will be around $10 \mathrm{~mL} / \mathrm{kg}$ body weight.

\section{UCBC administration}

Inclusion criteria

Extreme preterm infants with absence of severe brain injury on neonatal cranial ultrasounds, performed in the first week of life.

\section{Exclusion criteria}

Extreme preterm infants with evidence of severe preterm brain injury as defined by grade III-IV $\mathrm{IVH}^{20}$ and/or cystic periventricular leukomalacia. Infants who are likely to have redirection of intensive care due to any reason, as decided by the treating clinical team would also be excluded.

\section{Intervention}

UCBCs from autologous cord blood will be administered intravenously to infants, between D9 and D15 of life. This will follow a routine D8 cranial ultrasound scan. Infants with an active bacterial infection (blood culture positive in last 48 hours) or instability as determined by the treating team will have cell administration deferred, for administration, if possible, within the treatment window (D9-D15).

\section{Dose}

UCBCs will be administered intravenously (through a peripheral intravenous catheter) at a dose of 25-50 million viable cells $/ \mathrm{kg}$ body weight, 25 million/ $\mathrm{kg}$ being the proposed minimum dose and 50 million/ $\mathrm{kg}$ being the maximum dose. The dose will depend on the number of UCBCs able to be processed and deemed viable after cord blood collection. This dose is based on preclinical $^{78101521}$ and clinical studies ${ }^{316}$ on UCBC use for perinatal brain injury. If the number of cells available is less than 25 million $/ \mathrm{kg}$, then cell administration will not occur in this trial but cell characteristics will be studied. These extreme premature infants frequently receive blood products (packed red blood cells, plasma and rarely platelets at volumes of $10-15 \mathrm{~mL} / \mathrm{kg}$, and hence it was decided that the volume of cell infusion in this study will also be around $10 \mathrm{~mL} / \mathrm{kg}$. The cell administration protocol will be based on an ongoing current placental stem cell study at Monash Newborn. ${ }^{22}$

\section{Cell infusion protocol}

Infants will receive an infusion of UCBCs via a peripheral venous catheter. The suspension with UCBCs will be filtered through an inline intravenous filter. The infusion will be administered over $30 \mathrm{~min}$ via a vertical syringe pump. No other medications, blood products or solutions will be infused via the same peripheral intravenous site during the UCBC infusion.

\section{Primary outcomes}

The primary aims of this study are to test (1) feasibility of autologous cord blood collection and cell retrieval following processing from extremely premature infants; and (2) safety of autologous UCBC administration in eligible extremely preterm infants.

Feasibility will be determined by agreement to participate, ability to collect and process sufficient cord blood and then access to sufficient UCBCs within the second week of life to meet the infusion criteria. UCBCs will be collected from all potentially eligible infants but will only be administered to eligible infants as per inclusion and exclusion criteria above.

Safety of UCBC administration will be determined by occurrence of adverse events as defined below. Infants will be monitored as follows.

\section{Monitoring during UCBC infusion and nursery stay}

Infants will be observed for 2 hours prior to UCBC infusion to determine their baseline cardiorespiratory status and establish acceptable parameters for fluctuations during the infusion. During the infusion, HR, RR (Respiratory Rate), ECG, $\mathrm{SpO}_{2}$ (peripheral oxygen saturation) will be monitored continuously while blood pressure (BP), temperature and site of infusion will be checked every 15 min. Post infusion, HR, RR, ECG, $\mathrm{SpO}_{2}$ will be continuously monitored, while $\mathrm{BP}$ and temperature will be checked hourly.

Infants, as a result of their prematurity, are likely to remain inpatients in intensive and special care nurseries for at least 3-4 months following the UCBC administration. The routine clinical care afforded infants over this time will serve as monitoring for adverse events.

Routine care will include continuous cardiorespiratory monitoring; physical examination (daily while infants 
remains on respiratory support); anthropometry (weekly weight, head circumference, length); documentation of respiratory support requirements; chest radiograph, as clinically indicated; blood gas analysis, as determined by clinical team; cranial ultrasound, as per clinical practice but a minimum of two cranial ultrasounds post infusion prior to discharge (D28, D42); term equivalent age MRI brain, as per clinical practice.

\section{Monitoring post discharge}

Following discharge from nursery, infants will be assessed at 6, 12, 18 and 24 months corrected age. Assessment will focus on general health including growth parameters and physical examination, reporting of any adverse events and medication usage. Neurodevelopmental assessment will be performed using standardised assessment tools, including General Movements and Hammersmith Infant Neurological Examination (at 3 months corrected age), and the Bayley Scales of Infants and Toddler Development (IV edition) at 2 years corrected age.

\section{Defining adverse events}

Adverse events will be defined as follows:

- During infusion

- Local site reaction (erythema, oedema, extravasation at site of peripheral intravenous catheter site).

- Any sustained change of $30 \%$ or more from baseline in vital signs (HR, RR, BP, SpO2, Temp).

- Within first 24 hours of infusion

- Any event requiring cardiopulmonary resuscitation.

- Escalation of respiratory support (intubation of an infant receiving non-invasive respiratory support; or change to high frequency oscillatory ventilation in an infant receiving conventional ventilation at the time of UCBC infusion).

- Fluid bolus or initiation/escalation of inotropic support.

- Infection within 48 hours of UCBC infusion (culture proven bacterial, fungal or viral infection, or culture negative, clinically suspected infection).

\section{Data Safety Monitoring Board (DSMB)}

An independent DSMB has been formed comprising a neonatologist, and a cell scientist/biologist to review all adverse incidents. Adverse incidents will be reported to DSMB within 1 week of occurrence.

\section{Reporting to Human Research Ethics Committee (HREC)}

The following reports will be submitted to HREC:

- Any adverse event/reaction deemed suitable by DSMB to temporarily halt the trial pending review.

- An interim report after UCBC administration to 10 babies.

- Annual research progress report.

- Any updates to protocol/Participant Information and Consent Form (PICF).

\section{Secondary outcomes}

Neonatal and long-term general health and neurological outcomes, including cranial ultrasound changes after D8 of life (although unlikely to impact IVH outcomes as IVH is more a complication in the first week of life), term equivalent MRI brain findings (if clinically indicated), early neurodevelopment assessments in the first 3 months of corrected age (General Movements, Hammersmith Neonatal/Infant Neurological Examinations) and 2-year neurodevelopment assessment (Bayley Scales of Infant Development, s of Infant Development, BSID-IV). The secondary outcomes of the study participants may also be compared with a matched historical cohort (same criteria of no evidence of severe brain injury by $\mathrm{D} 8 \mathrm{scan}$ ) of extremely premature infants.

In a substudy, we will characterise the composition, characteristics and functionality of the preterm UCBCs collected from all enrolled infants. Comparative studies with stored term UCBCs collected for other studies will be considered.

We will also conduct a targeted cytokine analysis on infant's serum; namely interleukin 1b (IL-1b), IL-6, (Tumor Necrosis Factor) TNF- $\alpha$ and IL-10 at time (just before) of UCBC administration, 1 day post administration and at 36-37 weeks postconceptional age as potential biomarkers for response to cell therapy. These cytokines have been shown to be impacted by UCBC administration in previous studies. ${ }^{89}$

\section{Statistical methods}

As this is a phase I trial, detailed statistical analysis will not be required, but descriptive and inferential statistical analysis will be conducted as appropriate. Comparative analysis will be performed between study participants and historical matched controls.

\section{ETHICS AND DISSEMINATION \\ Ethics}

The study will be conducted in compliance with the approved protocol/amendment(s), conditions of Monash Health HREC approval and the NHMRC National Statement on Ethical Conduct in Human Research 2007 (updated May 2018). Expert neonatal clinical review panel and hospital ethics committee deliberated with the research team to determine the inclusion criteria. It was felt due to lack of acceptability by clinicians, and in the absence of safety evidence of UCBC transplantation in extremely premature infants; the trial should first include infants without severe brain injury to establish feasibility and safety. A patient consumer representative was involved in the ethics preparation and review of this protocol.

\section{Consent}

Fully informed and written consent will be obtained antenatally from the parents after detailed discussion regarding the 'experimental' nature of this study, and 
potential lack of benefits of cord blood collection and/ or UCBC administration. Consent will not be obtained during active labour or after birth. In all cases, written consent will be obtained using a specifically designed PICF. Participation will be voluntary and withdrawal is possible at any stage. Should withdrawal occurs after an infant receives the intervention, safety monitoring will be offered in line with the monitoring outlined in the protocol.

\section{Patient and public involvement}

Consumer input and feedback through the hospital ethics committee and CP Alliance (a patient advocacy non-profit organisation) consumer groups was sought in the design of this and other cord blood trials. A summary report will be made available to all participant families.

The outcomes of this study will be disseminated via peerreviewed journals and presented at scientific conferences.

\section{Twitter Atul Malhotra @Atul_Monash}

Contributors All authors contributed to the design of the study. AM drafted the initial protocol and manuscript. IN, SLM and GJ made critical revisions and edited the manuscript. All authors approved the final manuscript.

Funding Cell collection, processing, storage and release will be conducted by Cell Care Australia.

Competing interests None declared.

Patient and public involvement Patients and/or the public were involved in the design, or conduct, or reporting, or dissemination plans of this research. Refer to the Methods section for further details.

Patient consent for publication Not required.

Provenance and peer review Not commissioned; externally peer reviewed.

Open access This is an open access article distributed in accordance with the Creative Commons Attribution Non Commercial (CC BY-NC 4.0) license, which permits others to distribute, remix, adapt, build upon this work non-commercially, and license their derivative works on different terms, provided the original work is properly cited, appropriate credit is given, any changes made indicated, and the use is non-commercial. See: http://creativecommons.org/licenses/by-nc/4.0/.

ORCID iD

Atul Malhotra http://orcid.org/0000-0001-9664-4182

\section{REFERENCES}

1 Chow SSW, Creighton P, Kander V, et al. Report of the Australian and New Zealand neonatal network 2016. ANZNN, Sydney, 2018.

2 Bolisetty S, Dhawan A, Abdel-Latif M, et al. Intraventricular hemorrhage and neurodevelopmental outcomes in extreme preterm infants. Pediatrics 2014;133:55-62.
3 Cotten CM, Murtha AP, Goldberg RN, et al. Feasibility of autologous cord blood cells for infants with hypoxic-ischemic encephalopathy. $J$ Pediatr 2014;164:e971:973-9.

4 Hattori T, Sato Y, Kondo T, et al. Administration of umbilical cord blood cells transiently decreased hypoxic-ischemic brain injury in neonatal rats. Dev Neurosci 2015;37:95-104.

5 Aridas JDS, McDonald CA, Paton MCB, et al. Cord blood mononuclear cells prevent neuronal apoptosis in response to perinatal asphyxia in the newborn lamb. J Physiol 2016;594:1421-35.

6 Min K, Song J, Kang JY, et al. Umbilical cord blood therapy potentiated with erythropoietin for children with cerebral palsy: a double-blind, randomized, placebo-controlled trial. Stem Cells 2013;31:581-91.

7 Li J, Yawno T, Sutherland A, et al. Term vs. preterm cord blood cells for the prevention of preterm brain injury. Pediatr Res 2017;82:1030-8.

$8 \mathrm{Li} \mathrm{J}$, Yawno T, Sutherland A, et al. Preterm white matter brain injury is prevented by early administration of umbilical cord blood cells. Exp Neurol 2016;283:179-87.

9 Paton MCB, Allison BJ, Li J, et al. Human umbilical cord blood therapy protects cerebral white matter from systemic LPS exposure in preterm fetal sheep. Dev Neurosci 2018;40:258-70.

10 Malhotra A, Castillo-Melendez M, Allison BJ, et al. Neurovascular effects of umbilical cord blood-derived stem cells in growthrestricted newborn lambs : UCBCs for perinatal brain injury. Stem Cell Res Ther 2020;11:17.

11 McDonald CA, Penny TR, Paton MCB, et al. Effects of umbilical cord blood cells, and subtypes, to reduce neuroinflammation following perinatal hypoxic-ischemic brain injury. $J$ Neuroinflammation 2018;15:47.

12 Phillips AW, Johnston MV, Fatemi A. The potential for cell-based therapy in perinatal brain injuries. Trans/ Stroke Res 2013;4:137-48.

13 McDonald CA, Fahey MC, Jenkin G, et al. Umbilical cord blood cells for treatment of cerebral palsy; timing and treatment options. Pediatr Res 2018;83:333-44.

14 Jantzie LL, Scafidi J, Robinson S. Stem cells and cell-based therapies for cerebral palsy: a call for rigor. Pediatr Res 2018;83:345-55.

15 Li J, Yawno T, Sutherland AE, et al. Preterm umbilical cord blood derived mesenchymal stem/stromal cells protect preterm white matter brain development against hypoxia-ischemia. Exp Neurol 2018;308:120-31.

16 Yang J, Ren Z, Zhang C, et al. Safety of autologous cord blood cells for Preterms: a descriptive study. Stem Cells Int 2018;2018:1-9.

17 Ren Z, Xu F, Zhang X, et al. Autologous cord blood cell infusion in preterm neonates safely reduces respiratory support duration and potentially preterm complications. Stem Cells Trans/ Med 2020;9:169-76.

18 Wright C, Velickovic Z, Brown R, et al. Raising the standard: changes to the Australian code of good manufacturing practice (cGMP) for human blood and blood components, human tissues and human cellular therapy products. Pathology 2014;46:177-83.

19 International Netcord Foundation. NetCord-FACT international standards for cord blood collection, banking, and release for administration. 6 edn, 2016.

20 Papile LA, Burstein J, Burstein R, et al. Incidence and evolution of subependymal and intraventricular hemorrhage: a study of infants with birth weights less than 1,500 GM. J Pediatr 1978;92:529-34.

21 Paton MCB, Allison BJ, Fahey MC, et al. Umbilical cord blood versus mesenchymal stem cells for inflammation-induced preterm brain injury in fetal sheep. Pediatr Res 2019;86:165-73.

22 Baker EK, Malhotra A, Lim R, et al. Human amnion cells for the prevention of bronchopulmonary dysplasia: a protocol for a phase I dose escalation study. BMJ Open 2019;9:e026265. 